\title{
Simulation of a destructive avalanche at Maseguchi, Japan
}

\author{
D. M. MCClung, \\ Departments of Civil Engineering and Geography, University of British Columbia, Vancouver, \\ B.C., Canada V6T 124 \\ S. KOBAYASHI AND K. IZUMI \\ Research Institute for Hazards in Snowy Areas, Niigata University, Niigata 950-21, Japan
}

\begin{abstract}
In 1986, a large avalanche destroyed 11 houses and killed 13 people at Maseguchi, Japan. Previous attempts to model the avalanche were based on the assumption that it was a powder avalanche consisting of snow particles suspended by air turbulence. In this paper, the avalanche is modelled as a dry flowing avalanche with a dense core of flowing material at the base. It is suggested that for a comprehensive explanation of the observed damage and the characteristics of the avalanche deposit, the assumption that the avalanche was a flowing avalanche is more appropriate. The comparison of model results from a flowing versus a powder avalanche is of general interest for avalanche zoning and design of structures in avalanche-threatened areas.
\end{abstract}

\section{INTRODUCTION}

On 26 January 1986 a large, destructive dry-snow avalanche struck the village of Maseguchi, Niigata Prefecture, Japan. The damage included 11 houses destroyed or damaged, 13 fatalities and 11 injured. The scale and steepness of the path, the depth of the slab fracture and the mechanical properties of the snow combined to produce an extreme event. The snow observations made near the time of the event, as well as the details of the path, provide enough information to enable calculations of avalanche speeds and estimates of impact pressures.

Dry-snow avalanches usually belong to one of two basic classes: powder avalanches and flowing avalanches. A powder avalanche is usually envisioned as one in which the majority of the snow particles are suspended by turbulence and deposition takes place by a slow settlement of the snow particles once turbulence dies. For a powder avalanche, the volume concentration of snow particles, $C$, must be low throughout the flow, not just in the upper portion of the flow. Such avalanches are observed to form only under special conditions and they are actually rarely observed (see discussion below). The model of Fukushima and Parker (1990) contains assumptions compatible with a true powder avalanche model, e.g. that $C \ll<1$. Ackermann and Shen (1978) describe how the assumption of a turbulent flow breaks down if the concentration of solids becomes more than a few per cent, whereupon particle collisions become more likely, turbulence cannot be supported in the interstitial air and particle suspension becomes less likely.

In contrast, a dry flowing avalanche is one in which momentum transfer is by inter-particle collision and friction between particles and the sliding surface (snow or ground). A dry flowing avalanche has a dense core at the bottom ( $C$ is less than, but of order 1$)$. In addition, a dry flowing avalanche is usually surrounded by a snow "dust" cloud of material suspended by turbulent eddies at the top of the flow: field observations clearly show this effect. Since the dust cloud obscures the view of the dense core, the appearance of a flowing avalanche and a powder avalanche during motion can be similar. Usually, observations of the avalanche deposit, the destructive effects of the avalanche or some kind of physical measurements during motion are required to distinguish between powder and flowing avalanches.

In this paper, the Maseguchi event is modelled as a flowing avalanche using the scaling model of McClung (1990). The results are compared with the powder avalanche model of Fukushima and Parker (1990) and field measurements by Kobayashi and Izumi (1989). It is shown that a flowing avalanche model is required to explain the observations comprehensively (debris deposition and impact forces). A short description is given of the expected differences between destructive effects and modelling assumptions for flowing and powder avalanches.

\section{DESGRIPTION OF THE MASEGUCHI AVALANCHE}

The Maseguchi avalanche released at $2300 \mathrm{~h}$ on 26 January 1986 on the side of Mount Gongen. The total distance travelled along the incline was more than $2000 \mathrm{~m}$ and the vertical fall was about $800 \mathrm{~m}$. Figure 1 shows the profile of the avalanche as a function of distance travelled 


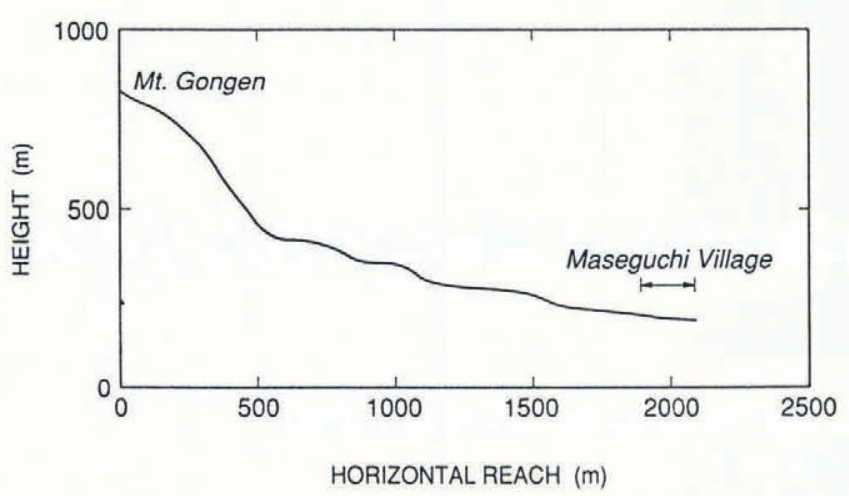

Fig. 1. Profile of the Maseguchi avalanche showing the location of the village (after Fukushima and Parker, 1990).

from the initiation point. The event began on an eastfacing slope and it gradually turned to the northeastfacing direction in which it struck the village at Maseguchi.

Kobayashi and Izumi (1989) reported snow properties at the townsite of Maseguchi measured four days after the avalanche (Fig. 2). The snow structure shows a dry layer of thickness in excess of $2 \mathrm{~m}$ overlying a fragile, weak, wet layer ( $7 \%$ water). It is expected that this profile is related to - but not equivalent to - conditions at the fracture site $800 \mathrm{~m}$ above.

Kobayashi and Izumi (1989) also reported field measurements of debris hardness and density (Fig. 2). The debris particles had an average density near $300 \mathrm{~kg} \mathrm{~m}^{-3}$ and they ranged from single crystals to balls $1-10 \mathrm{~cm}$ in diameter. According to the measurements published by Kobayashi and Izumi (1989), the depth of the deposit at Maseguchi was at least $1.3 \mathrm{~m}$. It was also determined from laboratory testing of wooden pieces of broken house pillar that the minimum impact force was 2 tonnes $\mathrm{m}^{-2}$.

\section{GHARACTERISTICS OF POWDER VERSUS FLOWING AVALANGHES}

Dry-snow avalanches in motion consist of particles (a mixture of ice and air) immersed in a fluid (air). The physical parameter which determines the crucial modelling assumptions and the friction at the bed is the volume

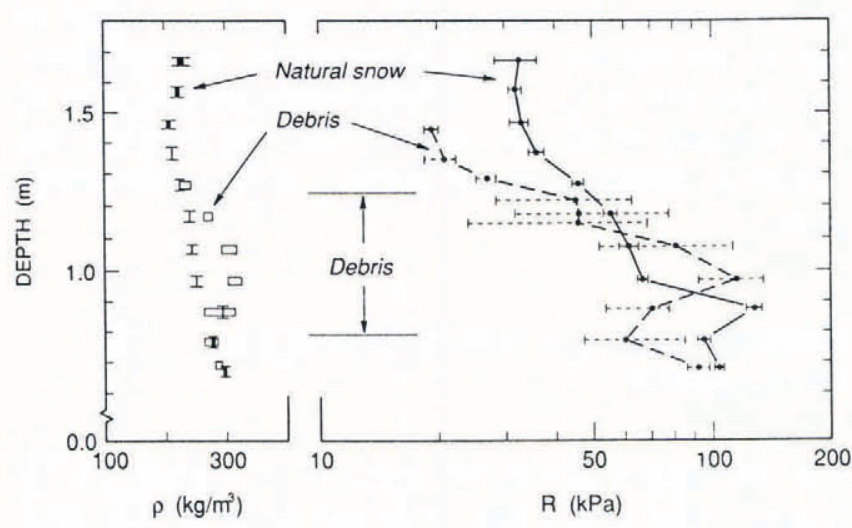

Fig. 2. Snowpack and debris density at the Maseguchi townsite. Density $\rho\left(\mathrm{kg} \mathrm{m}^{-3}\right)$ and hardness $R(\mathrm{kPa})$ (measured with the ram penetrometer) are shown. concentration of particles, $C$. If $C<<1$, then turbulence can be supported in the air between the particles and modelling might be approximated as a turbulent fluid; in this case the avalanche is called a powder avalanche. For a flowing avalanche, the mean free path between the particles is small and particle collisions or enduring friction contacts account for momentum transfer within the mass. In the case of flowing avalanches, the main friction component is at the sliding surface (bottom) of the avalanche and hence shear and normal forces should be strongly coupled there. Therefore, the modelling principles, forms of the frictional motion resistance and expected impact pressures may vary greatly for these two different classes of dry avalanche flows. Powder avalanches are expected to have relatively low density and quite low impact pressures. Flowing avalanches have relatively high density, fairly high speeds and high impact pressures; these characteristics combine to produce large destructive effects for flowing avalanches (de Quervain, 1966).

The characteristics of avalanche deposits are quite different for powder and flowing avalanches. For a powder avalanche, since the volume concentration of solids is in the order of a few per cent, the flow consists of a dilute suspension and the material settles over a broad area once internal turbulence dies. In the flowing avalanche, the dense core at the base stops fairly abruptly and the deposit consists of a collection of particles of different sizes in a well defined mass.

True powder avalanches, according to our field experience, occur in rather specialized conditions and, therefore, observations and data are quite rare. However, one of us (DMM) has often observed powder avalanches originating from ice falls on glaciers. Usually, the large particles of ice drop out quickly once the slope angle declines to about $10^{\circ}$ or less and the powder (or dust) cloud travels on for a much longer distance, often with minimal destructive effects. Our personal field observations and experiences show that it is possible for a person to be struck by such an avalanche and still survive. We believe this is mainly possible because of the low density of the flowing material.

Another example of low density flows from our field observations (DMM), is release of dry, loose snow avalanches from steep terrain. When the surface conditions are right, these avalanches may entrain additional snow on their descent and run for long distances. Our observations confirm that low density flows with most of the snow suspended in turbulent eddies do exist, but that they form under restricted conditions and massive deposits are not observed. McClung and Schaerer (1985) reported an impact pressure record and measured speed for an event approximating a powder avalanche with a flow density estimate of about 10 times that of air. We believe, however, that our field observations and measurements of these events should not be discounted.

\section{SGALING AND GALCULATIONS OF SPEEDS FOR THE MASEGUGHI AVALANGHE}

The observations of deposit characteristics, destructive effects and snow profile data (Kobayashi and Izumi, 
1989) provide the only information available to class the Maseguchi avalanche as a powder or flowing avalanche. All three of these information sources strongly indicate that the avalanche was a dry flowing avalanche. In this section, speeds are scaled according to a model by McClung (1990) and the results are compared with the powder avalanche model of Fukushima and Parker (1990).

Since the Maseguchi avalanche is an extreme event it is appropriate to apply the extreme event scaling model of McClung (1990). The runout distance (path length) for the avalanche is known from field observations to be in the middle of Maseguchi village about $2.2 \mathrm{~km}$ from the starting zone. McClung (1990) scaled maximum avalanche speeds for more than 60 avalanches and it was determined that an approximate upper limit for maximum speed is $V_{0} \sim 1.5 \sqrt{S_{0}}$ where $S_{0}$ is total path length traversed; for $S_{0}=2200 \mathrm{~m}$ this estimate gives $V_{0} \sim 70 \mathrm{~m} \mathrm{~s}^{-1}$.

In order to apply the scaling model to calculate the speed profile along the incline, the mean value of basal granular friction, $\bar{\mu}$, must be determined once the runout distance and path geometry are known. Also, an estimate must be made for the air (turbulent) drag at the top of the flow. For the Maseguchi event, the angle from the start to runout position is defined by $\tan \alpha=0.56\left(\alpha=22.5^{\circ}\right)$. The value of the air drag coefficient is given by McClung (1990) as

$$
D_{0}=\left(\frac{\rho_{\mathrm{t}}}{\bar{\rho}}\right) \frac{C_{\mathrm{f}}}{h},
$$

where $\rho_{t}$ is density at the top of the flow and $\bar{\rho}$ is the mean value of density in the flow. From McClung (1990), the ratio $\rho_{t} / \bar{\rho}$ is assumed to be approximately 0.1 . In Equation (1), $C_{\mathrm{f}}$ is turbulent drag coefficient at the top of the flow and $h$ is mean flow depth. For the Maseguchi event, a minimum value for $h$ is probably near $2 \mathrm{~m}$ and a value for the ratio $C_{\mathrm{f}} / h$ is near $0.006 \mathrm{~m}^{-1}$ (see e.g. McClung, 1990). This gives an approximate value $D_{0}^{-1}=3333 \mathrm{~m}$.

From the granular flow scaling model of McClung (1990), the value of the friction coefficient, $\mu$, at the bottom of the avalanche is approximated as a function of path length $S$ as

$$
\mu=\mu_{0}+\left(\bar{\mu}-\mu_{0}\right)(n+1)\left(\frac{S}{S_{0}}\right)^{n},
$$

where $\mu_{0}$ is the initial value, $n$ is determined by path steepness, and $S_{0}$ is known from the runout distance. From McClung (1990), $\mu$ and $n$ are given as a function of path steepness. The mean value of $\mu$ is given by

$$
\bar{\mu}=\tan \alpha\left(1-K_{0}\right),
$$

where $K_{0}$ is a constant: either 0 if no air drag is assumed or it may be determined by a numerical iterative procedure once $D_{0}$ and the avalanche stop position are specified (see e.g. McClung, 1990).

Figure 3 gives speed profile estimates for the Maseguchi avalanche modelled as an extreme event with stop position given as the middle of the village. The maximum speed $V_{\mathrm{m}}$ is estimated as $74 \mathrm{~ms}^{-1}$ at a path length $700 \mathrm{~m}$. This estimate is slightly higher for the ratio $V_{\mathrm{m}} / \sqrt{S_{0}}(=1.6)$ than the value 1.5 estimated from scaled

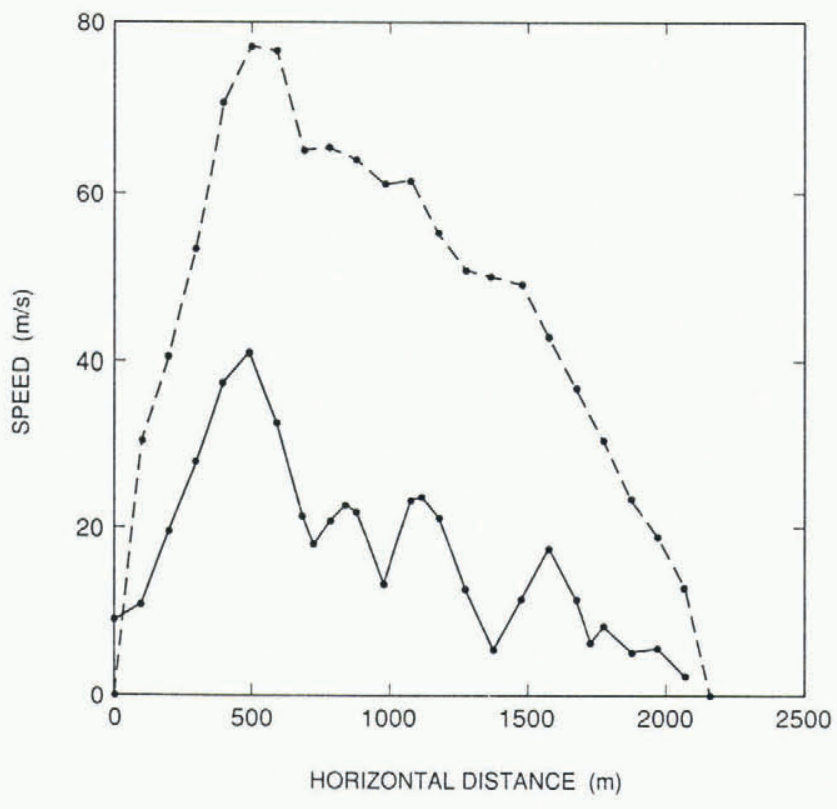

Fig. 3. Speed versus horizontal distance predicted by two models. - - flowing avalanche model (McClung, 1990); - powder avalanche model (Fukushima and Parker, 1990).

speed data by McClung (1990). The Maseguchi avalanche profile has a very steep section with slope angles near $50^{\circ}$ below the starting zone, which forces very high speeds in the model.

Figure 3 also shows the speed profile estimated by the powder avalanche model of Fukushima and Parker (1990), whose assumptions seem fairly realistic for a powder avalanche. It may be noted that their powder avalanche model gives an approach speed of about $7 \mathrm{~m} \mathrm{~s}^{-1}$ when the avalanche strikes the village, whereas the flowing avalanche model yields an approach speed of $31 \mathrm{~m} \mathrm{~s}^{-1}$. These differences have profound implications for destructive effects.

\section{ESTIMATE OF IMPACT FORCES}

Impact forces may be estimated as the product of flow density and speed squared. The flow density, $\rho$, of a mixture of snowballs and air may be estimated as

$$
\rho=\rho_{\mathrm{s}} C+\rho_{\mathrm{a}}(1-C)
$$

where $\rho_{\mathrm{B}}$ is the density of the debris materials (a mixture of ice and air) and $\rho_{\mathrm{a}}$ is the density of air.

For a powder avalanche, the calculations of Fukushima and Parker (1990) give $C=0.02$ as the avalanche approaches Maseguchi, and from Kobayashi and Izumi (1989) $\rho_{\mathrm{s}} \sim 300 \mathrm{~kg} \mathrm{~m}^{-3}$ and $\rho_{\mathrm{a}} \sim 1 \mathrm{~kg} \mathrm{~m}^{-3}$. With these the flow density becomes $\rho=7 \mathrm{~kg} \mathrm{~m}^{-3}$ and the estimated impact pressure, $I$, approximates to $\bar{\rho} v^{2}=0.34$ tonnes $\mathrm{m}^{-2}$; this may be compared with the minimum value estimated from laboratory testing of house pillars which approximates to 2 tonnes $\mathrm{m}^{-2}$. If the particles are taken as small, single ice crystals, $\bar{\rho} \sim 19 \mathrm{~kg} \mathrm{~m}^{-3}$ and $I \sim 0.9$ tonnes $\mathrm{m}^{-2}$.

For dry flowing avalanches, McClung and Schaerer (1985) estimated from impact pressures that $C \sim 0.35$ 
which gives $\bar{\rho}=110 \mathrm{~kg} \mathrm{~m}^{-3}$ for the Maseguchi avalanche. Using this value, and the approach speed $\left(31 \mathrm{~m} \mathrm{~s}^{-1}\right)$ gives $I=11$ tonnes $\mathrm{m}^{-2}$ at the bottom of the avalanche and it is assumed that $\rho_{\mathrm{t}} \simeq 0.1 \bar{\rho}$ at the top (see Equation (1)) to give $I \sim 1.1$ tonnes $\mathrm{m}^{-2}$ at the top of the avalanche. These values bracket the minimum estimates of Kobayashi and Izumi and therefore the model provides estimates which are the right order of magnitude in this case. Perla and Martinelli (1976) estimate pressures of 3 tonnes $\mathrm{m}^{-2}$ will destroy wood frame structures and 10 tonnes $\mathrm{m}^{-2}$ will uproot mature spruce trees. It seems that the 11 tonnes $\mathrm{m}^{-2}$ estimated will be in the correct range to explain the destruction at Maseguchi.

\section{ANALYSIS OF SPEEDS OF FLOWING AND POW- DER AVALANCHES}

Consider a simple analysis of a powder avalanche using the assumptions of Fukushima and Parker (1990) who assumed that the friction of the top and bottom of the avalanche is of the form $K V^{2}$ where $K$ is the drag coefficient times density appropriate for the top or bottom of the avalanche. Let $K_{\mathrm{t}}$ denote the value at the top and $K_{\mathrm{b}}$ that at the bottom. For simplicity, we ignore longitudinal gradients within the flow so that our analysis is consistent with a center-of-mass model.

By the work-energy theorem, the kinetic energy of the avalanche at maximum speed must equal the total work done by external forces over the path length to that point $S_{\mathrm{m}}$ (path length to maximum speed). Let $M_{0}$ be the mass and $V_{0}$ now be the maximum speed. The work done by external forces is given by

$$
W=\int_{0}^{S_{\mathrm{m}}} F \mathrm{~d} S
$$

where $S$ is path length and $F(S)$ is given by

$$
F(S)=M(S) g \sin \psi-K_{\mathrm{t}} V^{2}-K_{\mathrm{b}} V^{2},
$$

where $M(S)$ is mass, $\psi$ is local slope angle and $g$ is acceleration due to gravity. Equation (6) may be derived from the analysis in Appendix B of McClung (1990) by assuming shear and normal forces are not coupled at the lower boundary of the avalanche: instead, drag there is assumed to be due to turbulent, speed dependent resistance. Equating kinetic energy and work done by external forces gives

$$
\begin{aligned}
& \frac{1}{2} M_{0} V_{0}^{2}= \\
& \int_{0}^{S_{\mathrm{m}}} M(S) g(\sin \psi) \mathrm{d} S-\int_{0}^{S_{\mathrm{m}}} K_{\mathrm{t}} V^{2} \mathrm{~d} S-\int_{0}^{S_{\mathrm{m}}} K_{\mathrm{b}} V^{2} \mathrm{~d} S .
\end{aligned}
$$

From Figure 3, $\sin \psi=-\mathrm{d} y / \mathrm{d} S$ so that Equation (7) may be restated as

$$
V_{0}^{2}=2 \frac{\bar{M}}{M_{0}} g H_{0}-2 \frac{F_{\mathrm{t}}}{M_{0}}-2 \frac{F_{\mathrm{b}}}{M_{0}},
$$

where now $H_{0}$ is the vertical drop to the position of maximum speed, $\bar{M}$ is the mean value of the mass over $S_{\mathrm{m}}$, and $F_{\mathrm{t}}$ and $F_{\mathrm{b}}$ stand for the integrated top and bottom friction, respectively.
Consider now a simple model of mass entrainment: mass increasing linearly with $y$ over the interval 0 to $H_{0}$. If $M_{\mathrm{i}}$ is initial avalanche mass then

$$
M(y)=M_{\mathrm{i}}\left(1+\frac{N y}{H_{0}}\right),
$$

where $N$ is a measure of the mass gain. In Equation (9), if $N=1$ then the avalanche mass doubles by entrainment over the interval and if $N=9$, the mass increases by a factor of 10 over the interval. Integration of the first term of Equation (7) gives the first term of Equation (8):

$$
g H_{0}\left(\frac{N+1}{N+2}\right) \text {. }
$$

Upon substitution, Equation (8) becomes

$$
V_{0}^{2}=g H_{0}\left(\frac{N+2}{N+1}\right)-2 \frac{F_{\mathrm{t}}}{M_{0}}-2 \frac{F_{\mathrm{b}}}{M_{0}} .
$$

Consider some extreme limits from Equation (10): (1) if friction is ignored and there is no entrainment $(N=0), V_{0}=4.4 \sqrt{H_{0}}$ (equivalent to a free falling mass); (2) if $N=1$ (mass doubles) and friction is ignored, $V_{0}=3.8 \sqrt{H_{0}}$; (3) if $N=9$ (mass increases by a factor of 10 ) with friction ignored, $V_{0}=3.3 \sqrt{H_{0}}$. For the Maseguchi avalanche, the calculations of Fukushima and Parker (1990) and our own indicate $H_{0} \sim 500 \mathrm{~m}$ to give $V_{0} \sim 74 \mathrm{~m} \mathrm{~s}^{-1}$ in the latter case (factor of 10 increase in mass) with $85 \mathrm{~m} \mathrm{~s}^{-1}$ if the mass doubles. Maximum speed calculated by Fukushima and Parker is near $40 \mathrm{~m} \mathrm{~s}^{-1}$ : this shows the strong effect of the friction terms predicted in Equation (10).

In an earlier paper (McClung, 1990) data were presented for more than 70 flowing avalanches. These data show that an upper envelope of maximum speeds may be represented by $V_{0} \simeq 2.6 \sqrt{H}$, where $H$ is total vertical drop (about $700 \mathrm{~m}$ for Maseguchi, implying $\left.V_{0} \sim 70 \mathrm{~m} \mathrm{~s}^{-1}\right)$. From our calculations and those of Fukushima and Parker, $H / H_{0} \sim 1.4$ to give $V_{0} \sim 3.1 \sqrt{H_{0}}$. Therefore, given the assumptions above, it seems that the maximum speed of a powder avalanche at Maseguchi is at most comparable to that expected for flowing avalanches at the site. Calculations with realistic values of friction (Fukushima and Parker, 1990) predict a much lower maximum speed for the Maseguchi event when it is modelled as powder avalanche as opposed to a flowing avalanche. Their calculations show the importance of the two friction terms in Equation (10).

\section{DISCUSSION}

The character of the deposit and the magnitude of the destructive effects at Maseguchi strongly suggest the event was a flowing avalanche. Comparison of the estimated impact pressures from powder and flowing avalanche models tends to show that the flowing avalanche model is the more realistic choice in this case. It should be remarked, however, that our chosen model (McClung, 1990) is based only on approximate scaling, and therefore our conclusions with respect to impact forces support but do not validate this model. The model is proposed only within the scope of what is known about avalanche dynamics and speeds and, as such, its predictive 
capabilities are only order of magnitude estimates which seem consistent with this example.

The Maseguchi event is unique in that an avalanche with great destructive effects has been previously simulated using a realistic model of a powder avalanche. Comparison of the real destructive effects and those implied by the powder model shows that the minimum impact pressures for the Maseguchi event are more than two to five times those implied by the powder model of Fukushima and Parker (1990). The calculations by Fukushima and Parker are in agreement with field observations of true powder avalanches, i.e. destructive effects are usually much less than for flowing avalanches. One reason for this is that the implied flow densities are an order of magnitude less for a true powder avalanche than for a flowing avalanche (de Quervain, 1966; McClung and Schaerer, 1985). In order for material to remain suspended by turbulence, the flow density must be of order $10 \mathrm{~kg} \mathrm{~m}^{-3}$; otherwise, the volume concentration becomes too high, the assumption $C<<1$ becomes invalid, and a powder model is no longer applicable. In order for implied impact pressures (at maximum speed) to be equivalent for flowing and powder avalanches, the implied maximum speeds must be about three times higher for powder avalanches than for flowing avalanches. Speed data from flowing avalanches and our simplified theoretical analysis give some indication that the ratio of maximum speeds is comparable at most; this seems to confirm de Quervain's assertion that the destructive effects of flowing avalanches are greater than from powder avalanches.

From an engineering point of view, the most important aspect of a powder avalanche is that the dust cloud (greater than $10 \mathrm{~m}$ ) may extend higher than the dense core of a flowing avalanche (usually 2-5m). Therefore, when structures are planned in avalanchethreatened areas, the destructive effects of the powder cloud must be dealt with when considering either dry flowing avalanches in their top portion or powder avalanches.

\section{ACKNOWLEDGEMENTS}

This paper is a joint contribution of the Departments of Civil Engineering and Geography of the University of
British Columbia and the Institute for Hazards in Snowy Areas, Niigata University. This research was partly sponsored by the Institute for Research in Construction, National Research Council of Canada and the Natural Sciences and Engineering Council of Canada.

\section{REFERENCES}

Ackermann, N.L. and H.T. Shen. 1978. Flow of granular material as a two-component system. In US-Japan seminar: Continuum Mechanical and Statistical Approaches in the Mechanics of Granular Materials. Preprint. U.S. National Science Foundation and Japan Society for the Promotion of Science, 5-9 June, 1978, Sendai, Japan, 9-16.

Fukushima, Y. and G. Parker. 1990. Numerical simulation of powder-snow avalanches. J. Glaciol., 36(123), 229-237.

Kobayashi, S. and K. Izumi. 1989. A brief review of the Masequchi avalanche in Japan in 1986. In Proceedings of the International Snow Science Workshop, October 12-15, 1988, Whistler, BC. Vancouver, Canadian Avalanche Association, 57-62.

McClung, D. M. 1990. A model for scaling avalanche speeds. 7. Glaciol., 36(123), 188-198.

McClung, D. M. and P.A. Schaerer. 1985. Characteristics of flowing snow and avalanche impact pressures. Ann. Glaciol., 6, 9-14.

Norem, H., F. Irgens and B. Schieldrop. 1987. A continuum model for calculating snow avalanche velocities. International Association of Hydrological Sciences Publication 162 (Symposium at Davos 1986 - Avalanche Formation, Movement and Effects), 363-379.

Perla, R. I. and M. Martinelli, Jr. 1976. Avalanche handbook. Washington, DC, U.S. Department of Agriculture. (Handbook 489.)

Quervain, M.R. de. 1966. Problems of avalanche research. International Association of Scientific Hydrology Publication 69 (Symposium at Davos 1965 - Scientific Aspects of Snow and Ice Avalanches), 15-22.

Turner, J.S. 1973. Buoyancy effects in fluids. Cambridge, Cambridge University Press.

The accuracy of references in the text and in this list is the responsibility of the authors, to whom queries should be addressed. 